\title{
Effects of Curcumin Treatment in a Diabetic Neuropathic Pain Model of Rats: Involvement of c-Jun N-Terminal Kinase Located in the Astrocytes and Neurons of the Dorsal Root Ganglion
}

\author{
Hanwool Park, Jong-Hyuk Lee, Ji Hoon Sim, Jihoon Park, Seong-Soo Choi $\mathbb{D}$, \\ and Jeong Gil Leem \\ Department of Anesthesiology and Pain Medicine, University of Ulsan College of Medicine, Asan Medical Center, Seoul, \\ Republic of Korea \\ Correspondence should be addressed to Seong-Soo Choi; choiss@amc.seoul.kr
}

Received 22 July 2020; Revised 6 November 2020; Accepted 8 January 2021; Published 19 January 2021

Academic Editor: Marcelo M. Valença

Copyright ( 92021 Hanwool Park et al. This is an open access article distributed under the Creative Commons Attribution License, which permits unrestricted use, distribution, and reproduction in any medium, provided the original work is properly cited.

\begin{abstract}
Curcumin (diferuloylmethane) is a major component of turmeric, which is isolated from the rhizomes of Curcuma longa L. from the family Zingiberaceae. It is used as a dietary pigment for curry and in traditional Indian medicine for its anti-inflammatory and attenuating pain effects. This study aimed to evaluate the beneficial effects of curcumin in a rat model of diabetic neuropathic pain. Additionally, we investigated the involvement of the phosphorylated form of c-Jun N-terminal kinase (pJNK) located in the neurons and astrocytes of the dorsal root ganglion (DRG). To induce diabetic neuropathic pain in rats, $50 \mathrm{mg} / \mathrm{kg}$ of streptozotocin (STZ) was intraperitoneally injected. After 4 weeks, rats were administered the vehicle, $10 \mathrm{mg} / \mathrm{kg} / \mathrm{day}$ curcumin, or $50 \mathrm{mg} / \mathrm{kg} / \mathrm{day} \mathrm{curcumin}$ orally for 4 consecutive weeks. One day after the final drug administration, we performed behavioral tests to measure responses of rats to mechanical, heat, cold, and acetone-induced cold stimuli. After behavioral tests, pJNK expression in the DRG was evaluated using western blot assay and immunohistochemistry. Curcumin treatment for 4 consecutive weeks in STZ-induced diabetic neuropathic pain rats improved behavioral responses to mechanical, cold, and thermal stimuli. Increased pJNK expression in the astrocytes and neurons of the DRG in STZ-induced diabetic neuropathic pain rats was reduced by curcumin treatment for 4 consecutive weeks. We suggest that curcumin can be an option for the treatment of diabetes-related neuropathic pain, and one of the mechanisms that underlie the action of curcumin may involve pJNK expression in the astrocytes and neurons of the DRG.
\end{abstract}

\section{Introduction}

Neuropathic pain is one of the most common complications of diabetes mellitus (DM) and an estimated one-third of patients with DM have painful diabetic neuropathy [1]. It is characterized by hyperalgesia (increased sensitivity to pain), allodynia (pain sensation to nonpainful stimulation), dysesthesia (an unpleasant sense of touch), and paresthesia (abnormal sensation without a cause) and is caused by either peripheral nerve damage or changed neuronal signaling. However, molecular and cellular mechanisms that underlie neuropathic pain are not fully understood.

Curcumin (diferuloylmethane) is a natural product from the Curcuma longa L. from the family Zingiberaceae. It is a member of the curcuminoid family and used as a dietary pigment in curry and an ancient medicine for many purposes including asthma, allergy, anorexia, coryza, cough, hepatic disease, and sinusitis. Nowadays, curcumin is known to play an important role in inflammatory conditions. Curcumin decreases postsurgical allodynia [2], mitigates diabetic neuropathic pain [3], prevents the development of diabetic neuropathy [4], and decreases pain associated with depression [5]. Currently, the main analgesic mechanism of curcumin is thought to involve signals such as tumor necrosis factor- $\alpha$ (TNF- $\alpha$ ), nitric oxide (NO) [6], and extracellular signal-regulated kinase (ERK) [7] in the antiinflammatory pathway. Also, there are other analgesic mechanisms involved in curcumin such as activation of 
NF- $\kappa \mathrm{B}[8]$, noradrenergic and serotoninergic system $\beta 2$ adrenoreceptor and 5-HT1A receptor [9], NO-cGMP-ATPsensitive $K+$ channel pathways [10], TTX-R sodium currents [11], and opioid system [12].

Similar to ERK, c-Jun N-terminal kinase (JNK) is a member of the mitogen-activated protein kinase (MAPK) family. It regulates neuronal functions, immunological actions, and embryonic development [13]. JNK is also activated in the neurons of the dorsal root ganglion (DRG) and spinal cord after the ligation of a spinal nerve resulting in neuropathic pain [14]. JNK is activated when it becomes phosphorylated (phosphorylated JNK (pJNK)) [13]. Interestingly, JNK is also one of the most important kinases implicated in hyperglycemia and the pathogenesis of diabetes [15].

Curcumin is known to inhibit JNK activation [16-18]. However, it is unknown whether curcumin affects JNK in the neurons and astrocytes of the DRG. DRG is important for communication between the peripheral and the central nervous systems, and it is a target for pain management [19]. Therefore, the present study investigated the effects of curcumin on diabetic neuropathic pain induced by streptozotocin (STZ) in rats and on pJNK expression in the astrocytes and neurons of the DRG.

\section{Materials and Methods}

This study was approved by the Institutional Animal Care and Use Committee of the Asan Institute for Life Sciences, Asan Medical Center (approval number 2012-13-211). The committee follows the guidelines of the Institute of Laboratory Animal Resources. We performed all experiments according to the ethical guidelines of the International Association for the Study of Pain [20]. The animals were euthanized painlessly after the completion of all the experiments.

2.1. Experimental Animals. Male Sprague-Dawley rats $(200 \sim 250 \mathrm{~g})$ were housed in a standard cage under a 12-hour light-dark cycle. Humidity and temperature were controlled at $65 \pm 5 \%$ and $21 \pm 1^{\circ} \mathrm{C}$, respectively. Water and food were freely provided. The animals were allowed to habituate to the laboratory environment for at least 2 hours before performing behavioral experiments.

\subsection{Generation of the Diabetic Neuropathic Pain Model and} the Experiment Protocol. Diabetic neuropathic pain can be easily induced by the systemic administration of STZ [21-24]. Usually, rats can manifest diabetic neuropathy 4 weeks after a single administration of STZ $[22,25]$.

As shown in the graphical abstract (Figure 1), rats were randomly assigned to six groups ( $n=10$ per group): (1) control (Ctr) + vehicle (Veh), (2) Ctr $+10 \mathrm{mg} / \mathrm{kg} /$ day of curcumin $(\mathrm{Ctr}+\mathrm{CMN} 10)$, (3) $\mathrm{Ctr}+50 \mathrm{mg} / \mathrm{kg} /$ day of curcumin (Ctr + CMN50), (4) STZ + Veh, (5) STZ + CMN10, and (6) STZ + CMN50. Initially, saline solution at $0.9 \%$ (Ctr) or $50 \mathrm{mg} / \mathrm{kg}$ of STZ was injected intraperitoneally (IP). After 4 weeks, rats were also orally treated with $10 \mathrm{mg} / \mathrm{kg} /$ day curcumin, dissolved in 5\% alcohol and 5\% dextrose in water (5DW), and either $50 \mathrm{mg} / \mathrm{kg} /$ day $\mathrm{CMN}$ or vehicle (Veh, $5 \mathrm{DW})$ for 4 consecutive weeks. One day after the final drug administration, we performed behavioral tests to measure the responses of rats to mechanical, heat, cold, and acetoneinduced cold stimuli. After behavioral tests, we performed western blot assay and immunohistochemistry for pJNK in the DRG. The Ctr + CMN10 and STZ + CMN10 groups were excluded from western blot analysis and immunohistochemistry because the CMN50 groups showed better behavioral effects of curcumin than the CMN10 groups.

2.3. Curcumin and Antibodies. We used curcumin (Santacruz, Dallas, Texas, USA, \#sc-200509) in this study. Glial fibrillary acidic protein (GFAP, Cell Signaling Technology, Danvers, USA, \#3670, 1:100), pJNK (Cell Signaling Technology, Danvers, USA, \#5668, 1:50 for IHC, 1:1000 for western blot), Neurofilament 200 (NF200, Sigma-Aldrich, St. Louis, Missouri, USA, N5389, $1: 40$ ), and $\beta$-actin (BETHYL, Montgomery, Texas, USA, A300-491, $1: 10000$ ) were used as primary antibodies. BETHYL A120-101D3, A90-116D2, and A120-101P were used as secondary antibodies.

2.4. Behavioral Tests. Behavioral tests were performed to measure responses to mechanical, heat, cold, and acetoneinduced cold stimuli. All experiments were performed during the light phase $(10: 00-16: 00)$.

Mechanical allodynia was tested using a Von Frey filament (Stoelting, Wood Dale, IL). The plantar surface of the hindfoot was tested. We used eight types of calibrated von Frey filaments $(0.41 \mathrm{~g}, 0.70 \mathrm{~g}, 1.20 \mathrm{~g}, 2.00 \mathrm{~g}, 3.63 \mathrm{~g}, 5.50 \mathrm{~g}$, $8.50 \mathrm{~g}$, and $15.10 \mathrm{~g}$ ). We pressed the plantar surface of the feet for 6 seconds with these filaments. Rapid withdrawal or startling was measured as a positive response. The 50\% withdrawal threshold was measured by using the up-down method [26].

Cold allodynia against chemical stimuli was tested using the acetone [27]. A drop of acetone was applied to the midplantar surface of the hind paw for five times. The frequency of the paw withdrawals in response to the acetone was measured.

Allodynia and hypersensitivity to thermal and cold stimuli were analyzed using a hot/cold plate apparatus (Ugo Basile, Comerico, Italy). For thermal allodynia and hypersensitivity testing, the hot plate temperature was adjusted to $42 \pm 0.1^{\circ} \mathrm{C}$ and $50 \pm 0.1^{\circ} \mathrm{C}$, respectively [28]. For cold allodynia and hypersensitivity testing, the cold plate temperature was adjusted to $10 \pm 0.1^{\circ} \mathrm{C}$ and $4 \pm 0.1^{\circ} \mathrm{C}$, respectively [28]. The latency to first hind paw withdrawal or licking was measured. Every rat was tested three times. The cut-off time for allodynia was 30 seconds for the thermal test and 100 seconds for the cold test [29]. The cut-off time for hypersensitivity was 15 seconds for both thermal and cold tests [28].

2.5. Western Blot. After the final behavioral test, DRG tissues were isolated for western blot analysis. Tissues were washed twice with cold Tris-buffered saline and lysed with $2 \%$ SDS 
(a)

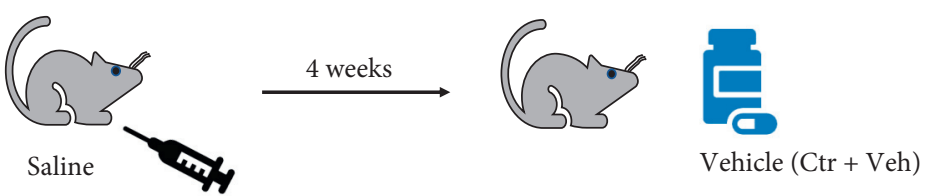

(b)
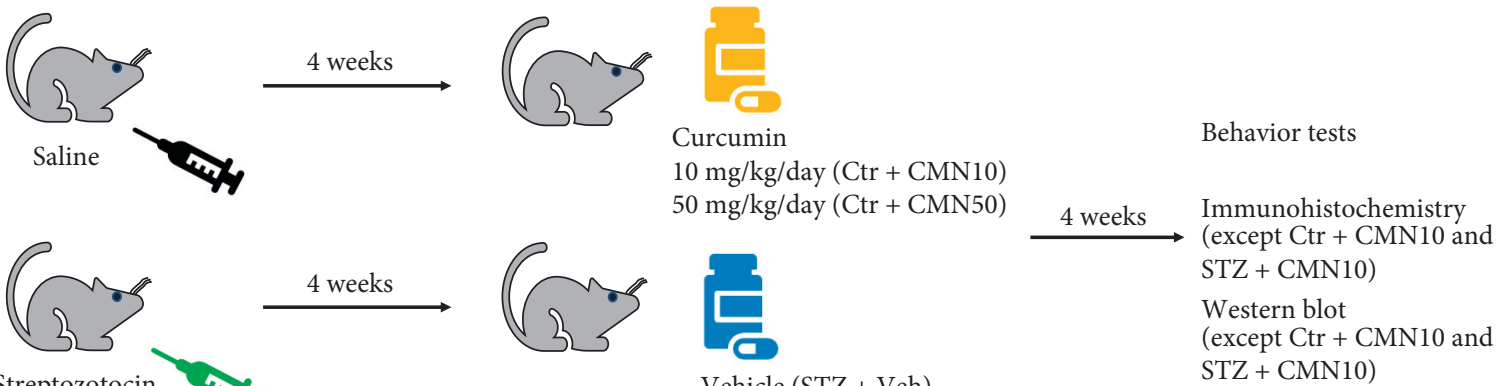

(c)
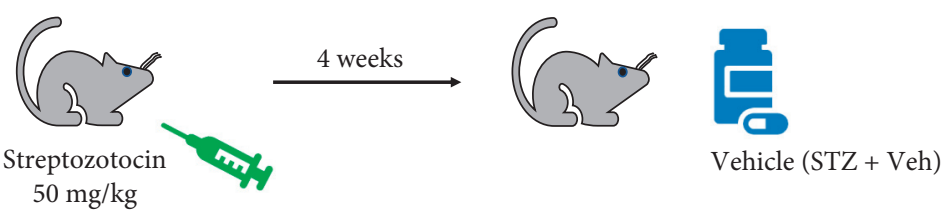

$\mathrm{STZ}+\mathrm{CMN10)}$

Western blot

$\mathrm{STZ}+\mathrm{CMN} 10)$

(d)
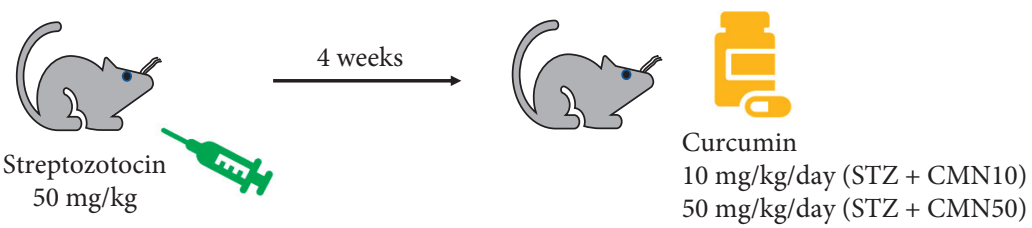

FIgURE 1: Simple experimental scheme. (a, b) Groups of rats were injected with saline solution at $0.9 \%$ intraperitoneally (IP). Four weeks later, they received either vehicle $(\mathrm{Ctr}+\mathrm{Veh}), 10 \mathrm{mg} / \mathrm{kg} / \mathrm{day}(\mathrm{Ctr}+\mathrm{CMN} 10)$, or $50 \mathrm{mg} / \mathrm{kg} /$ day of curcumin $(\mathrm{Ctr}+\mathrm{CMN} 50)$ orally for 4 consecutive weeks. The experiments were subsequently conducted. (c, d) Groups of rats were injected with $50 \mathrm{mg} / \mathrm{kg}$ of streptozotocin (STZ) IP. Four weeks later, they received vehicle $(\mathrm{STZ}+\mathrm{Veh})$, curcumin $10 \mathrm{mg} / \mathrm{kg} / \mathrm{day}(\mathrm{STZ}+\mathrm{CMN} 10)$, or $50 \mathrm{mg} / \mathrm{kg} / \mathrm{day}(\mathrm{STZ}+\mathrm{CMN} 50) \mathrm{orally}$ for 4 weeks before the start of the experiments.

lysis buffer containing $0.1 \mathrm{mM} \mathrm{Na}_{3} \mathrm{VO}_{4}, 3 \mathrm{mg} / \mathrm{mL}$ aprotinin, and $20 \mathrm{mM} \mathrm{NaF}$. Because the DRG of a single rat was not sufficient for western blotting, we mixed samples from six rats for each group. After sonication, the protein concentration was measured using a detergent compatible protein assay reagent (Bio-Rad Laboratories, Hercules, California, USA). The total protein samples $(40 \mu \mathrm{g})$ were used for the blotting. After immunoblotting with the antibodies, the ECL-plus solution (Amersham Pharmacia Bioscience, Piscataway, New Jersey, USA) was used. The specific blotted signals for $\mathrm{pJNK}$ and $\beta$-actin were quantified using the ImageJ freeware (NIH, Betheseda, Maryland, USA).

2.6. Immunohistochemistry. After the completion of behavioral tests, we injected rats with zoletil $(60 \mathrm{mg} / \mathrm{kg})$ and xylazine $(10 \mathrm{mg} / \mathrm{kg})$ intraperitoneally to enable DRG sampling for immunostaining. We perfused rats with $4 \%$ paraformaldehyde via the left ventricle of the heart for the fixation. The DRG was isolated and fixed immediately in $4 \%$ paraformaldehyde. The fixed DRG samples were embedded in paraffin. We sectioned the DRG samples into $10 \mu \mathrm{m}$ thick sections using a microtome and mounted the sections onto slides. Deparaffinization was performed using xylene and ethanol. The blocking solution comprised 5\% donkey serum, $0.3 \%$ Triton X-100, and $1 \%$ bovine serum albumin in phosphate-buffered saline. After incubating overnight with GFAP, NF200, and pJNK antibodies, the tissues were incubated with secondary antibodies for 2 hours. We acquired images using a confocal microscope (LSM780, Carl Zeiss,
Obercochen, Germany) and used Image pro plus ver 5.1 imaging software (Media Cybernetics, Rockville, Maryland, USA) for analysis.

2.7. Statistical Analysis. Data are presented as means \pm standard error. Behavioral test data were evaluated using one-way analysis of variance (ANOVA) with Bonferroni post hoc test using GraphPad Prism Version 5.01 for Windows (GraphPad Software, San Diego, CA, USA). $p$ value $<0.05$ was considered as significant.

\section{Results}

3.1. Effects of Curcumin on STZ-Induced Diabetic Neuropathic Painful Responses in Rats: Behavioral Responses to Mechanical and Thermal Stimuli. We subjected the STZ-induced diabetic neuropathic pain rats to a battery of allodynia and hyperalgesia tests (Figure 2). STZ-induced diabetic neuropathic pain rats showed significantly reduced paw withdrawal threshold to mechanical stimuli. Consecutive treatment of diabetic neuropathic pain rats with curcumin for 4 weeks increased the withdrawal threshold to mechanical stimuli $(p<0.05)$ although the threshold was not fully recovered to the level of the control group (Figure 2(a)). STZ-induced diabetic neuropathic pain rats also exhibited increased frequency of paw withdrawal in response to acetone-induced cold stimuli. This increased frequency of the paw withdrawals induced by acetone in STZ-induced diabetic neuropathic pain rats significantly reduced following 


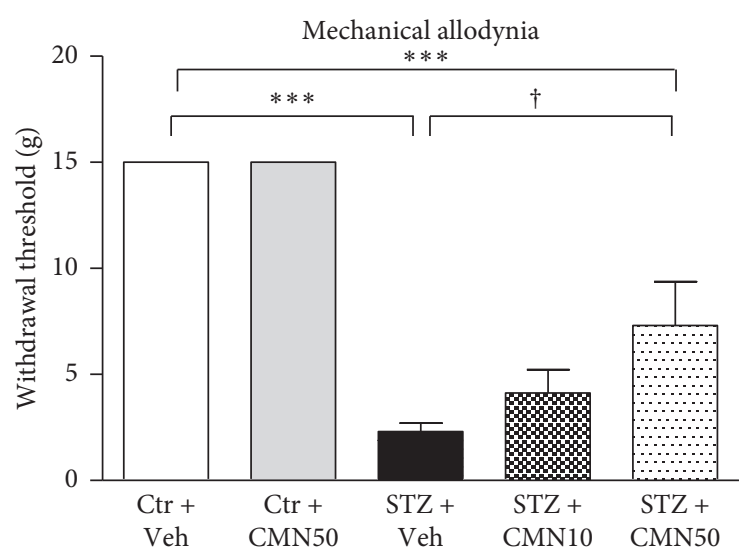

(a)

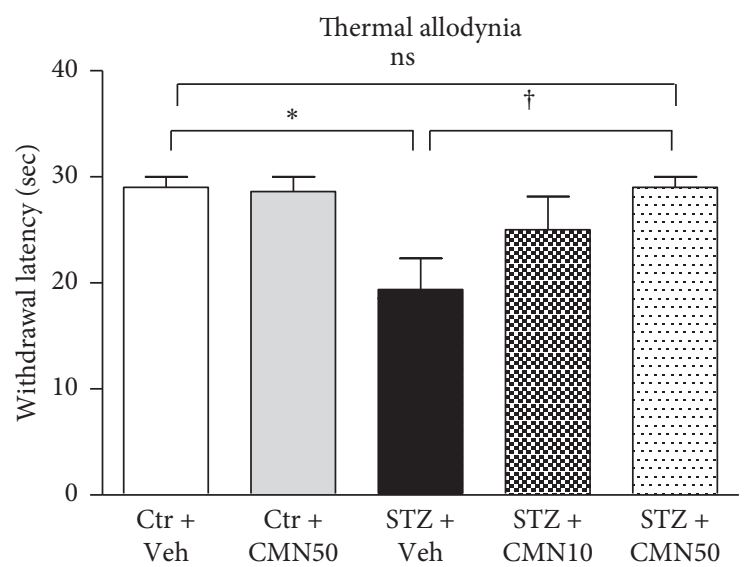

(c)

Cold allodynia

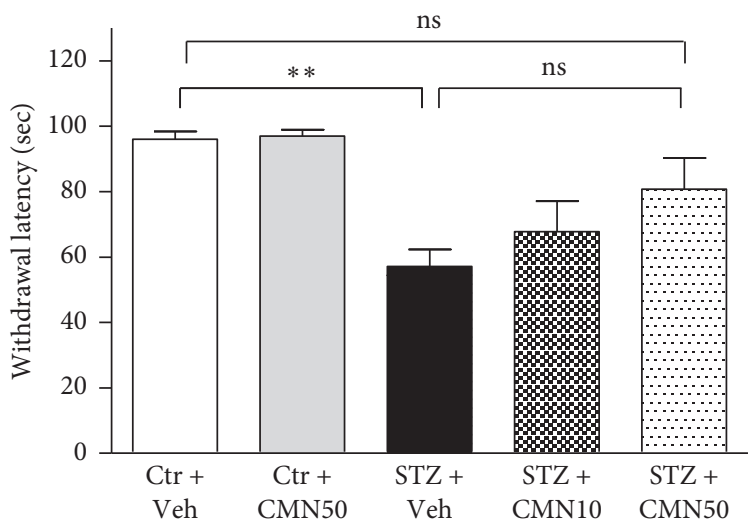

(e)

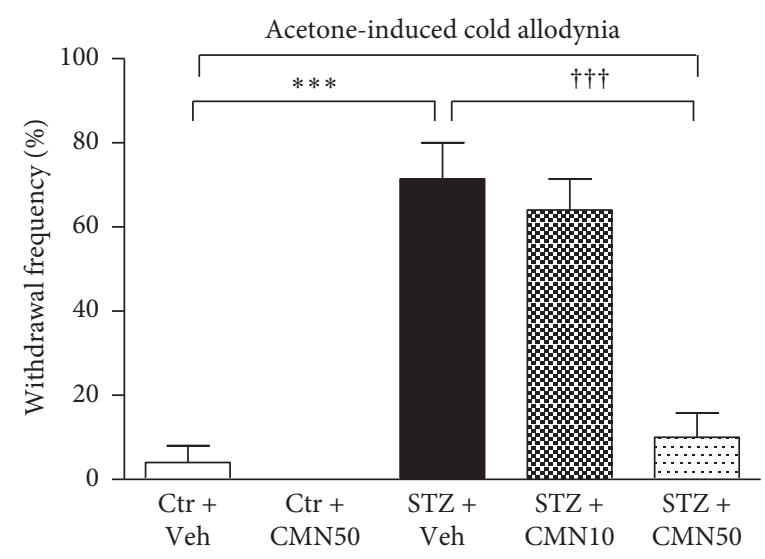

(b)

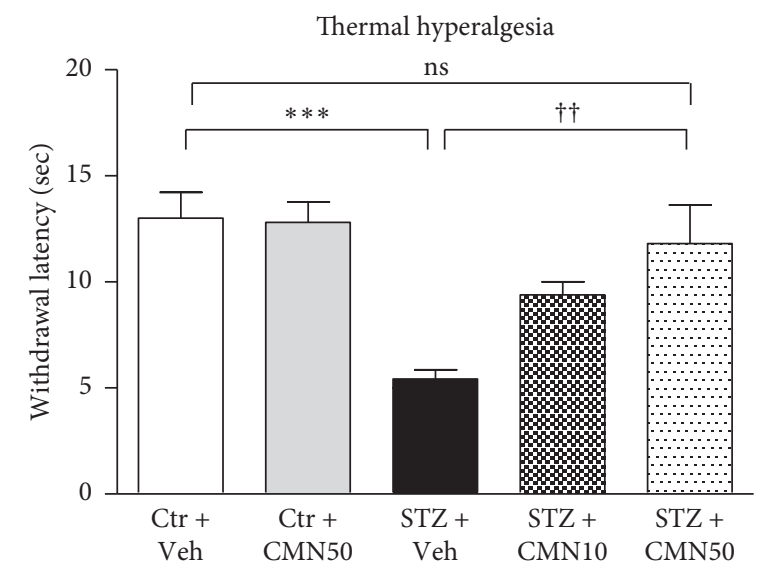

(d)

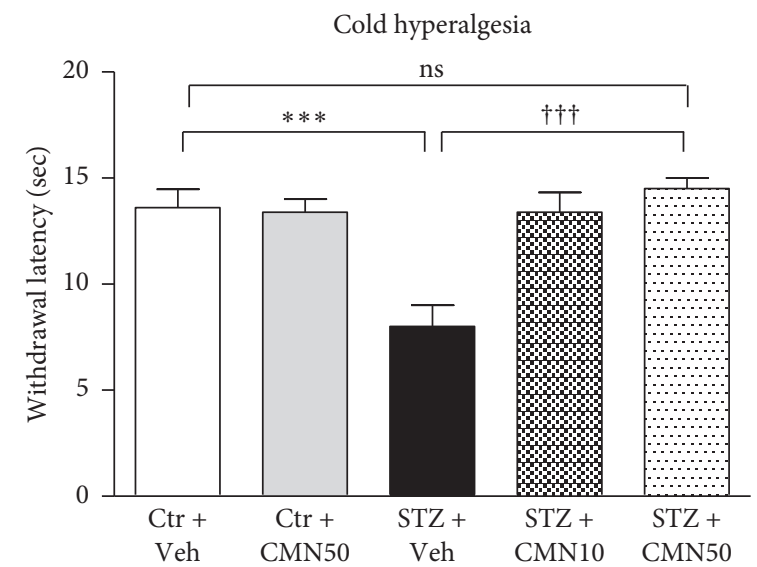

(f)

FIGURE 2: Effects of curcumin (CMN) administered orally on the diabetic neuropathic painful behavior induced by streptozotocin (STZ). STZ $(50 \mathrm{mg} / \mathrm{kg}$ ) was intraperitoneally injected to induce diabetic neuropathy in rats. After 4 weeks, rats were treated orally with vehicle and either $10 \mathrm{mg} / \mathrm{kg} /$ day or $50 \mathrm{mg} / \mathrm{kg} /$ day of $\mathrm{CMN}$ for 4 consecutive weeks. One day after final drug administration, behavioral tests were performed to measure responses to mechanical (a), acetone-induced cold (b), thermal ((c) $42^{\circ} \mathrm{C}$ and (d) $\left.50^{\circ} \mathrm{C}\right)$, and cold ((e) $10^{\circ} \mathrm{C}$ and (f) $\left.4{ }^{\circ} \mathrm{C}\right)$ stimuli ( $n=10$ animals per group, one-way ANOVA with Bonferroni post hoc). ${ }^{*} p<0.05,{ }^{* *} p<0.001$, and ${ }^{* * *} p<0.001$ compared to the $\mathrm{Ctr}+$ Veh group. ${ }^{\dagger} p<0.05,{ }^{\dagger \dagger} p<0.01$, and ${ }^{\dagger \dagger} p<0.001$ compared to the STZ + Veh group.

consecutive treatment with curcumin for 4 weeks (Figure 2(b), $p<0.001$ ). As shown in Figures 2(c) and 2(d), paw withdrawal latency to thermal stimuli $\left(42^{\circ} \mathrm{C}\right.$ and $\left.50^{\circ} \mathrm{C}\right)$ also significantly decreased in STZ-induced diabetic neuropathic pain rats. Consecutive treatment of diabetic neuropathic pain rats with curcumin for 4 weeks significantly reversed the decreased paw withdrawal latency to thermal stimuli (thermal allodynia, Figure 2(c), $p<0.05$; and 
thermal hyperalgesia, Figure 2(d), $p<0.01)$. Similarly, paw withdrawal latencies to cold stimuli $\left(10^{\circ} \mathrm{C}\right.$ and $\left.4^{\circ} \mathrm{C}\right)$ significantly decreased in STZ-induced diabetic neuropathic pain rats (Figure 2(e), $p<0.01$; Figure $2(\mathrm{f}), p<0.001$ ). Consecutive treatment of diabetic neuropathic pain rats with curcumin for 4 weeks reversed the decreased paw withdrawal latency to $10^{\circ} \mathrm{C}$ (Figure $2(\mathrm{e}), p>0.05$ ) and $4^{\circ} \mathrm{C}$ cold stimuli (Figure 2(f), $p<0.001$ ). Although the reversal of paw withdrawal latency to $10^{\circ} \mathrm{C}$ was not significant (Figure 2(e)), the tendency was prominent.

\subsection{Effects of Curcumin on pJNK Expression in STZ-Induced} Diabetic Neuropathic Pain Rats: Involvement of pJNK in the Astrocytes and Neurons of the DRG. Immunoblot analyses of the DRG revealed that the levels of pJNK protein expression were elevated in STZ-induced diabetic neuropathic pain rats (Figure 3). Consecutive treatment with $50 \mathrm{mg} / \mathrm{kg}$ curcumin for 4 consecutive weeks decreased the elevated pJNK expression induced by STZ administration. The treatment of sham control groups with curcumin did not change the amount of pJNK protein expressed.

Immunohistochemical analysis revealed an increase in pJNK immunoreactivity of DRG cells. These cells colocalized with GFAP (astrocyte marker) and NF200 (neuronal marker) positive cells, as shown in Figures 4(c) and 5(c), respectively. Consecutive treatment of STZ-induced diabetic neuropathic pain rats with $50 \mathrm{mg} / \mathrm{kg}$ curcumin for 4 weeks decreased the number of pJNK immunoreactive cells that were colocalized with both GFAP and NF200 in the DRG (Figures 4(d) and 5(d), respectively). Vehicle (control) or curcumin administration to the control group did not alter the pJNK immunoreactivity of the DRG cells (Figures 4(a) and 4(b) and 5(a)and 5(b), respectively).

\section{Discussion}

We examined the ability of curcumin to ameliorate neuropathic pain behaviors in STZ-induced diabetic neuropathic pain rats. Curcumin treatment decreased mechanical, cold, and thermal allodynia and hyperalgesia. Consistent with the findings of this study, previous studies also showed similar effects of curcumin on neuropathic pain. The treatment of STZ-induced diabetic neuropathic pain animals with curcumin reduced paw licking and tail-withdrawal response [3], decreased mechanical allodynia [12], improved sensorimotor deficits [12], and decreased thermal and mechanical hyperalgesia [30]. In another rat model of neuropathic pain from brachial plexus avulsion, administration of curcumin markedly increased mechanical withdrawal threshold and decreased paw-withdrawal frequency to cold stimuli [31]. Additionally, two clinical trials involving curcumin confirmed its beneficial effects on diabetic neuropathy $[32,33]$. Parallel, double-blind randomized, placebo-controlled trials showed that the treatment of patients with type $2 \mathrm{DM}$ complicated by peripheral neuropathy with nanocurcumin supplements reduced glycated hemoglobin ( $\mathrm{HbAlc}$ ), fasting blood sugar, total neuropathy score, total reflex score, temperature, depression, and anxiety scores [32, 33].
The mechanism of action of curcumin effect in diabetic neuropathy is currently under investigation. The pathogenesis of neuropathic pain is complex, making it difficult to identify the mechanism underlying the effect of curcumin on neuropathy. Diabetic neuropathy is thought to occur as a result of hyperglycemia and lack of neurotrophic support from insulin/C-peptide [34]. Hyperglycemia causes cytokine releases, oxidative stress, lipid peroxidation, and neuroinflammation $[35,36]$. Curcumin exhibited anti-inflammatory effects through the inhibition of TNF- $\alpha$, NO, ERK, and nuclear factor-kappa B $[6,7,34,37]$. Liu and coworkers have also showed that curcumin can decrease neuropathic pain by downregulation of spinal interleukin- $1 \beta$ via suppressing NAcht leucine-rich-repeat protein 1 inflammasome, and activation of Janus kinase 2-signal transducer and activator of transcription 3 cascade [38]. Furthermore, it has been reported that curcumin downregulates the activation of the JNK pathway in hyperglycemia models in vitro and in vivo. The in vitro model was induced by applying bisphenol A (BPA) into LO2 cells [16], while the in vivo model was induced using STZ followed by the examination of the spinal cord [18]. In western blot analysis of the DRG in the present study, curcumin treatment normalized the increased DRG pJNK expression induced by STZ administration. Because the DRG of a single rat was not sufficient for western blotting, we mixed samples from six rats for each group. Although we did not perform statistical analysis on the western blot results, the tendency toward a normalized pJNK expression might be enough to suggest that the analgesic effect of curcumin involves DRG pJNK protein expression in STZ-induced diabetic neuropathic pain rats.

In STZ-induced diabetic neuropathic pain models, pJNK is induced in the spinal cord neurons but not in the astrocytes [39]. However, they did not investigate which cell type located in the DRG expresses JNK although the immunoblot showed an increase in JNK levels of DRG in STZinduced diabetic neuropathic rats. JNK is also activated in the spinal astrocytes in other chronic pain conditions, including nerve injury induced by sciatic nerve ligation [14], inflammation induced by complete Freund's adjuvant [40], bone cancer [41], and skin cancer [42]. Additionally, JNK phosphorylation increased in the neurons of the DRG in an STZ-induced diabetic neuropathic pain animal model [43]. However, there has been no study on pJNK expression in the astrocytes in an STZ-induced diabetic neuropathic pain model. In the immunohistochemical analysis to determine DRG cells that expressed pJNK, we found that pJNK was activated not only in the neurons but also in the astrocytes of the DRG in STZ-induced diabetic neuropathic pain rats. The present study demonstrated that curcumin normalized pJNK expression in the astrocytes and neurons of the DRG in STZ-induced diabetic neuropathic pain rats. This suggests that curcumin administration decreases diabetic neuropathic pain responses by normalizing functional JNK activation in DRG cells in STZ-induced diabetic neuropathic pain rats. Additionally, the loss of A $\delta$ fibers leads to cold hyperalgesia, while the loss of $\mathrm{C}$ fibers leads to decreased thermal and mechanical pain thresholds [44]. Curcumin may differentially affect the aforementioned fibers. This may 


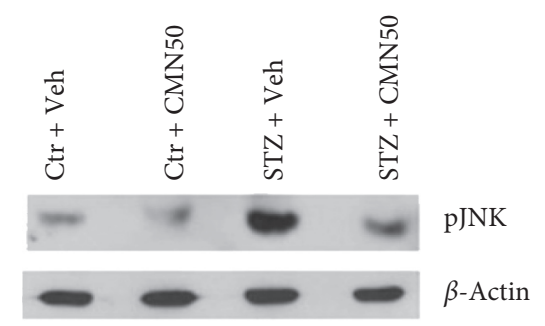

(a)

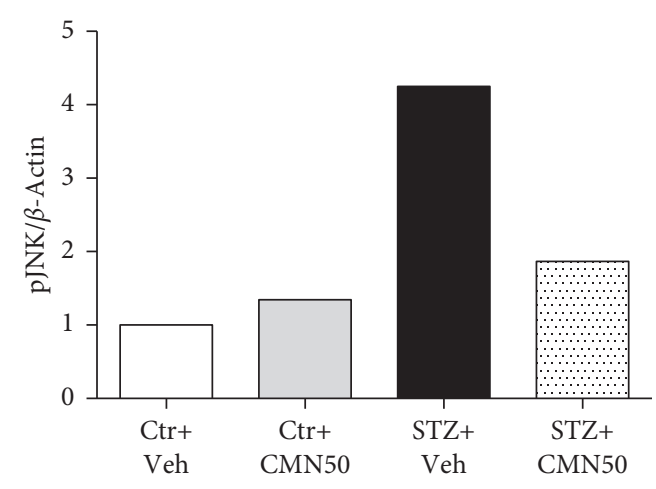

(b)

FIGURE 3: Western blot analysis of pJNK protein expression in the dorsal root ganglion (DRG). Total protein extraction from DRG was used for the western blot analysis. pJNK expression was increased in streptozotocin- (STZ-) induced diabetic neuropathic pain rats (STZ + Veh, $n=6$ ), which was subsequently normalized by treatment with $50 \mathrm{mg} / \mathrm{kg} /$ day of curcumin $(\mathrm{CMN})$ for 4 consecutive weeks $(\mathrm{STZ}+\mathrm{CMN} 50$, $n=6)$. $\beta$-Actin is a loading control.

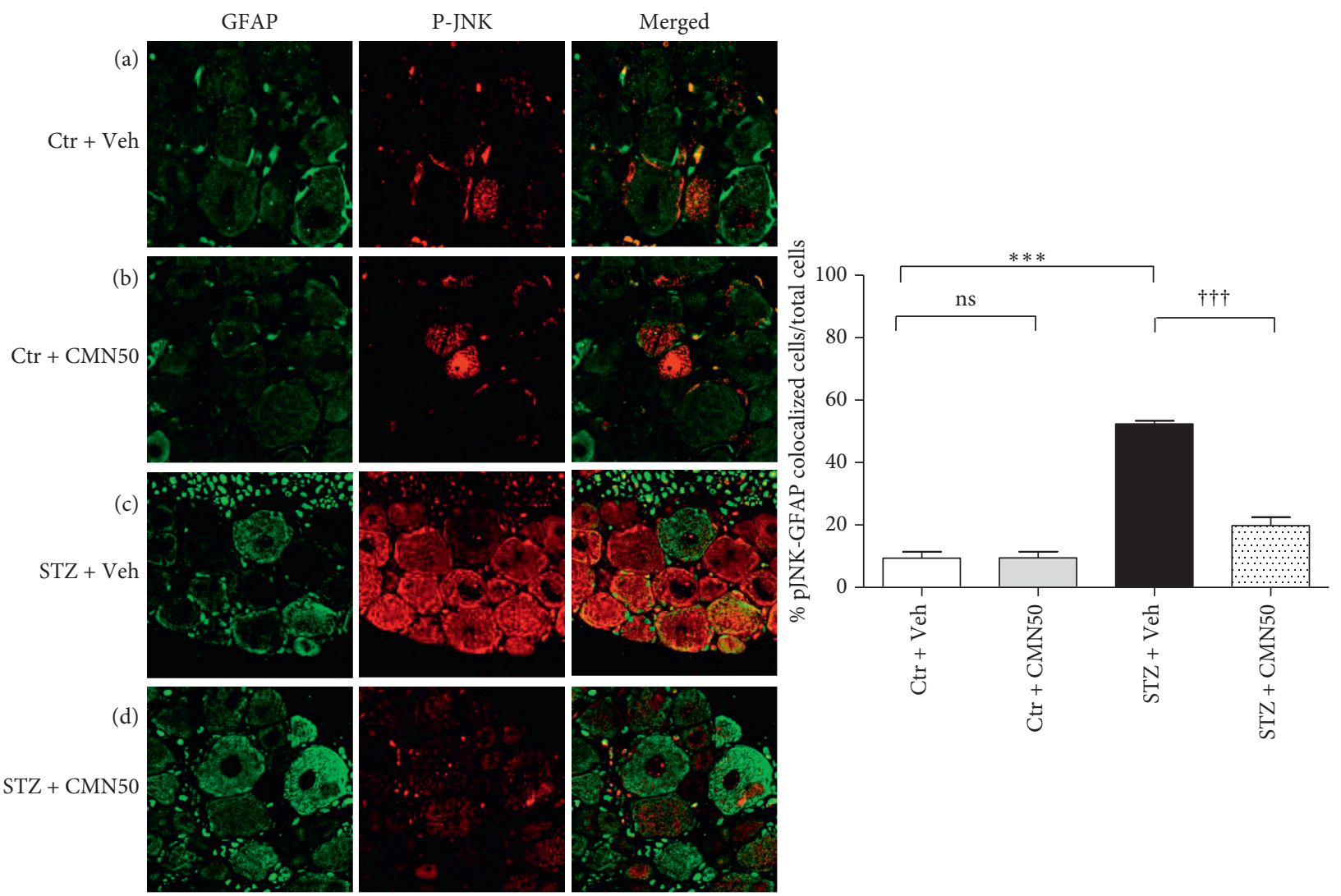

FIGURE 4: Immunohistochemistry for pJNK (red, pJNK antibody) expression in the astrocytes of the dorsal root ganglion (DRG) (green, GFAP antibody). Treatment of streptozotocin- (STZ-) induced diabetic neuropathic pain rats (STZ + CMN50) with $50 \mathrm{mg} / \mathrm{kg} / \mathrm{day}$ of curcumin (CMN) for 4 consecutive weeks normalized pJNK expression in the astrocyte of the DRG. (a) Control group (Ctr + Veh, $n=4)$; (b) curcumin-treated control group (Ctr + CMN50, $n=4)$; (c) STZ-induced diabetic neuropathic pain rats (STZ + Veh, $n=4)$; (d) curcumintreated STZ-induced diabetic neuropathic pain rats $(\mathrm{STZ}+\mathrm{CMN50,} n=4) .{ }^{* * *} p<0.001$ compared to the Ctr $+\mathrm{Veh}$ group; ${ }^{\dagger \dagger \dagger} p<0.001$ compared to the STZ+Veh group. 


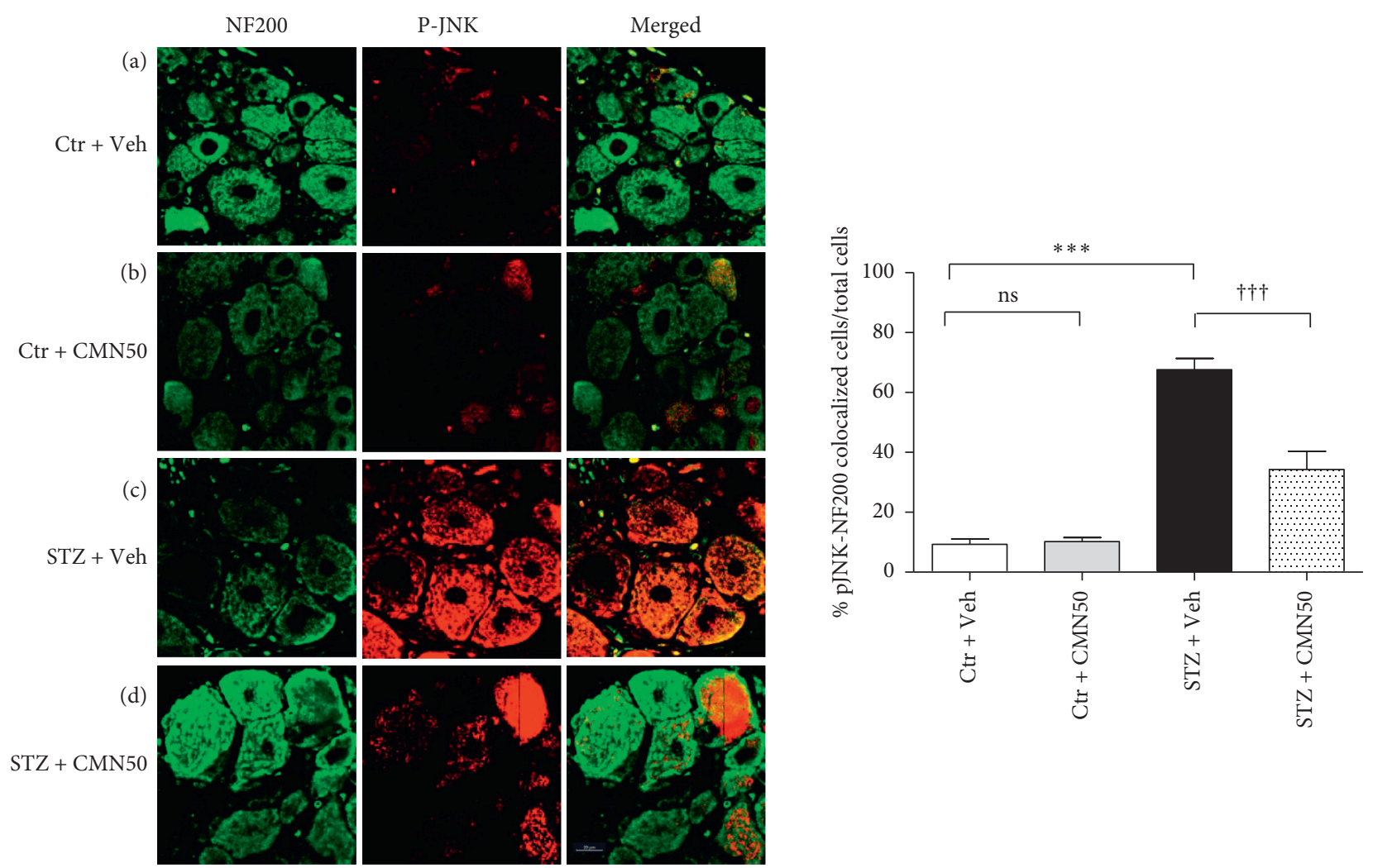

FIGURE 5: Immunohistochemistry for pJNK (red, pJNK antibody) expression in the neurons of the dorsal root ganglion (DRG) (green, NF200 antibody). Treatment of streptozotocin- (STZ-) induced diabetic neuropathic pain rats (STZ + CMN50) with $50 \mathrm{mg} / \mathrm{kg} / \mathrm{day}$ of curcumin (CMN) for 4 consecutive weeks normalized pJNK expression in the neurons of the DRG. (a) Control group (Ctr + Veh, $n=4)$, (b) curcumin-treated control group (Ctr $+\mathrm{CMN50}, n=4)$, (c) STZ-induced diabetic neuropathic pain rats (STZ + Veh, $n=4)$, and (d) curcumin-treated STZ-induced diabetic neuropathic pain rats $(\mathrm{STZ}+\mathrm{CMN50,} n=4) .{ }^{* * *} p<0.001$ compared to the Ctr $+\mathrm{Veh}$ group; ${ }^{\dagger \dagger} p<0.001$ compared to the STZ + Veh group.

explain why only mechanical allodynia was not fully improved. However, our immunohistochemical analysis could not differentiate between the effect of curcumin on $\mathrm{A} \delta$ fibers and that on $\mathrm{C}$ fibers.

\section{Conclusions}

We demonstrated the effect of curcumin in a rat model of diabetic pain induced by STZ. Curcumin may be useful in the management of diabetic neuropathic pain characterized by hyperalgesia or allodynia. In addition, we found that pJNK expression in the astrocytes and neurons of the DRG was increased by STZ-induced diabetic neuropathic rats. This increased pJNK expression might be decreased by curcumin treatment. We suggest that curcumin can be an option for the treatment of diabetes-related neuropathic pain and that one of the mechanisms of action of curcumin may involve pJNK expression in the astrocytes and neurons of the DRG.

\section{Data Availability}

The original data used to support the findings of this study are available from the corresponding author upon request.

\section{Conflicts of Interest}

The authors declare that they have no conflicts of interest.

\section{Authors' Contributions}

Hanwool Park and Jong-Hyuk Lee contributed equally to this work. Hanwool park did formal analysis and data curation, wrote the original draft, and visualized the study. Jong-Hyuk Lee validated the study, managed resources, wrote the original draft, reviewed and edited the manuscript, and administrated the project. Ji Hoon Sim and Jihoon Park performed data curation and validated the study. Jeong Gil Leem conceptualized, validated, and investigated the study, prepared methodology, managed resources, and reviewed and edited the manuscript. Seong-Soo Choi prepared methodology, analyzed using software, did formal analysis and data curation, reviewed and edited the manuscript, and investigated, visualized, and supervised the study.

\section{Acknowledgments}

This study was supported by a grant (2017-737) from the Asan Institute for Life Sciences and Corporate Relations (Yuhan Corporation) of Asan Medical Center (2015-673), Seoul, Korea. 


\section{References}

[1] C. A. Abbott, R. A. Malik, E. R. E. van Ross, J. Kulkarni, and A. J. M. Boulton, "Prevalence and characteristics of painful diabetic neuropathy in a large community-based diabetic population in the U.K," Diabetes Care, vol. 34, no. 10, pp. 2220-2224, 2011.

[2] K. A. Agarwal, C. D. Tripathi, B. B. Agarwal, and S. Saluja, "Efficacy of turmeric (curcumin) in pain and postoperative fatigue after laparoscopic cholecystectomy: a double-blind, randomized placebo-controlled study," Surgical Endoscopy, vol. 25, no. 12, pp. 3805-3810, 2011.

[3] S. Sharma, K. Chopra, and S. K. Kulkarni, "Effect of insulin and its combination with resveratrol or curcumin in attenuation of diabetic neuropathic pain: participation of nitric oxide and TNF-alpha," Phytotherapy Research, vol. 21, no. 3, pp. 278-283, 2007.

[4] H. N. Attia, N. M. Al-Rasheed, N. M. Al-Rasheed, Y. A. Maklad, A. A. E. Ahmed, and S. A. B. Kenawy, "Protective effects of combined therapy of gliclazide with curcumin in experimental diabetic neuropathy in rats," Behavioural Pharmacology, vol. 23, no. 2, pp. 153-161, 2012.

[5] V. Arora, A. Kuhad, V. Tiwari, and K. Chopra, "Curcumin ameliorates reserpine-induced pain-depression dyad: behavioural, biochemical, neurochemical and molecular evidences," Psychoneuroendocrinology, vol. 36, no. 10, pp. 1570-1581, 2011.

[6] S. Sharma, S. K. Kulkarni, J. N. Agrewala, and K. Chopra, "Curcumin attenuates thermal hyperalgesia in a diabetic mouse model of neuropathic pain," European Journal of Pharmacology, vol. 536, no. 3, pp. 256-261, 2006.

[7] F. T. Ji, J. J. Liang, L. Liu, M. H. Cao, and F. Li, "Curcumin exerts antinociceptive effects by inhibiting the activation of astrocytes in spinal dorsal horn and the intracellular extracellular signal-regulated kinase signaling pathway in rat model of chronic constriction injury," Chinese Medical Journal, vol. 126, no. 6, pp. 1125-1131, 2013.

[8] H. Cao, J.-W. Zheng, J.-J. Li, B. Meng, J. Li, and R.-S. Ge, "Effects of curcumin on pain threshold and on the expression of nuclear factor $\kappa \mathrm{B}$ and $\mathrm{CX} 3 \mathrm{C}$ receptor 1 after sciatic nerve chronic constrictive injury in rats," Chinese Journal of Integrative Medicine, vol. 20, no. 11, pp. 850-856, 2014.

[9] X. Zhao, Y. Xu, Q. Zhao, C.-R. Chen, A.-M. Liu, and Z.-L. Huang, "Curcumin exerts antinociceptive effects in a mouse model of neuropathic pain: descending monoamine system and opioid receptors are differentially involved," Neuropharmacology, vol. 62, no. 2, pp. 843-854, 2012.

[10] T. Pastrana-Quintos, G. Salgado-Moreno, J. Pérez-Ramos, A. Coen, and B. Godínez-Chaparro, "Anti-allodynic effect induced by curcumin in neuropathic rat is mediated through the NO-cyclic-GMP-ATP sensitive $\mathrm{K}(+)$ channels pathway," BMC Complementary Medicine and Therapies, vol. 20, no. 1, p. $83,2020$.

[11] B. Meng, L.-L. Shen, X.-T. Shi et al., "Effects of curcumin on TTX-R sodium currents of dorsal root ganglion neurons in type 2 diabetic rats with diabetic neuropathic pain," Neuroscience Letters, vol. 605, pp. 59-64, 2015.

[12] H. R. Banafshe, G. A. Hamidi, M. Noureddini, S. M. Mirhashemi, R. Mokhtari, and M. Shoferpour, "Effect of curcumin on diabetic peripheral neuropathic pain: possible involvement of opioid system," European Journal of Pharmacology, vol. 723, pp. 202-206, 2014.

[13] A. Zeke, M. Misheva, A. Reményi, and M. A. Bogoyevitch, "JNK signaling: regulation and functions based on complex protein-protein partnerships," Microbiology and Molecular Biology Reviews, vol. 80, no. 3, pp. 793-835, 2016.

[14] Z.-Y. Zhuang, Y. R. Wen, and D. R. Zhang, "A peptide c-Jun $\mathrm{N}$-terminal kinase (JNK) inhibitor blocks mechanical allodynia after spinal nerve ligation: respective roles of JNK activation in primary sensory neurons and spinal astrocytes for neuropathic pain development and maintenance," Journal of Neuroscience, vol. 26, no. 13, pp. 3551-3560, 2006.

[15] M. Igarashi, H. Wakasaki, N. Takahara et al., "Glucose or diabetes activates p38 mitogen-activated protein kinase via different pathways," Journal of Clinical Investigation, vol. 103, no. 2, pp. 185-195, 1999.

[16] S. Geng, S. Wang, W. Zhu et al., "Curcumin suppresses JNK pathway to attenuate BPA-induced insulin resistance in LO2 cells," Biomedicine \& Pharmacotherapy, vol. 97, pp. 15381543, 2018.

[17] W. Zhao, X. Zhou, G. Qi, and Y. Guo, "Curcumin suppressed the prostate cancer by inhibiting JNK pathways via epigenetic regulation," Journal of Biochemical and Molecular Toxicology, vol. 32, no. 5, p. e22049, 2018.

[18] Y.-S. Lee, D.-C. Cho, C. H. Kim, I. Han, E. Y. Gil, and K.-T. Kim, "Effect of curcumin on the inflammatory reaction and functional recovery after spinal cord injury in a hyperglycemic rat model," The Spine Journal, vol. 19, no. 12, pp. 2025-2039, 2019.

[19] L. Liem, E. van Dongen, F. J. Huygen, P. Staats, and J. Kramer, "The dorsal root ganglion as a therapeutic target for chronic pain," Regional Anesthesia and Pain Medicine, vol. 41, no. 4, pp. 511-519, 2016.

[20] M. Zimmermann, "Ethical guidelines for investigations of experimental pain in conscious animals," Pain, vol. 16, no. 2, 1983.

[21] W. J. Schnedl, S. Ferber, J. H. Johnson, and C. B. Newgard, "STZ transport and cytotoxicity. Specific enhancement in GLUT2-expressing cells," Diabetes, vol. 43, no. 11, pp. 1326-1333, 1994.

[22] J. S. Nam, Y. S. Cheong, M. H. Karm et al., "Effects of nefopam on streptozotocin-induced diabetic neuropathic pain in rats," The Korean Journal of Pain, vol. 27, no. 4, pp. 326-333, 2014.

[23] A. A. F. Sima and K. Sugimoto, "Experimental diabetic neuropathy: an update," Diabetologia, vol. 42, no. 7 , pp. 773-788, 1999.

[24] B. L. Furman, "Streptozotocin-induced diabetic models in mice and rats," Current Protocols in Pharmacology, vol. 70, pp. 41-45, 2015.

[25] K. K. Wu and Y. Huan, "Streptozotocin-induced diabetic models in mice and rats," Current Protocols in Pharmacology, vol. 40, no. 1, pp. 41-45, 2008.

[26] S. R. Chaplan, F. W. Bach, J. W. Pogrel, J. M. Chung, and T. L. Yaksh, "Quantitative assessment of tactile allodynia in the rat paw," Journal of Neuroscience Methods, vol. 53, no. 1, pp. 55-63, 1994.

[27] C. Yoon, Y. Y. Wook, N. H. Sik, K. S. Ho, and C. J. Mo, "Behavioral signs of ongoing pain and cold allodynia in a rat model of neuropathic pain," Pain, vol. 59, no. 3, pp. 369-376, 1994.

[28] I. Yalcin, A. Charlet, M.-J. Freund-Mercier, M. Barrot, and P. Poisbeau, "Differentiating thermal allodynia and hyperalgesia using dynamic hot and cold plate in rodents," The Journal of Pain, vol. 10, no. 7, pp. 767-773, 2009.

[29] W. U. Koh, S. S. Choi, and J. H. Kim, “The preventive effect of resiniferatoxin on the development of cold hypersensitivity induced by spinal nerve ligation: involvement of TRPM8," BMC Neuroscience, vol. 17, no. 1, p. 38, 2016. 
[30] T. Jia, J. Rao, and L. Zou, "Nanoparticle-encapsulated curcumin inhibits diabetic neuropathic pain involving the p2y12 receptor in the dorsal root ganglia," Frontiers in Neuroscience, vol. 11, p. 755, 2017.

[31] W. Xie, W. Xie, Z. Kang, C. Jiang, and N. Liu, “Administration of curcumin alleviates neuropathic pain in a rat model of brachial plexus avulsio," Pharmacology, vol. 103, no. 6, pp. 324-332, 2019.

[32] S. Asadi, M. S. Gholami, F. Siassi, M. Qorbani, and G. Sotoudeh, "Beneficial effects of nano-curcumin supplement on depression and anxiety in diabetic patients with peripheral neuropathy: a randomized, double-blind, placebocontrolled clinical trial," Phytotherapy Research, vol. 34, no. 4, pp. 896-903, 2020.

[33] S. Asadi, M. S. Gholami, F. Siassi, M. Qorbani, K. Khamoshian, and G. Sotoudeh, "Nano curcumin supplementation reduced the severity of diabetic sensorimotor polyneuropathy in patients with type 2 diabetes mellitus: a randomized double-blind placebo- controlled clinical trial," Complementary Therapies in Medicine, vol. 43, pp. 253-260, 2019.

[34] D. J. Daugherty, A. Marquez, N. A. Calcutt, and D. Schubert, "A novel curcumin derivative for the treatment of diabetic neuropathy," Neuropharmacology, vol. 129, pp. 26-35, 2018.

[35] G. M. Somfai, B. Knippel, É. Ruzicska et al., "Soluble semicarbazide-sensitive amine oxidase (SSAO) activity is related to oxidative stress and subchronic inflammation in streptozotocin-induced diabetic rats," Neurochemistry International, vol. 48, no. 8, pp. 746-752, 2006.

[36] Y. Panahi, N. Khalili, E. Sahebi et al., "Curcuminoids modify lipid profile in type 2 diabetes mellitus: a randomized controlled trial," Complementary Therapies in Medicine, vol. 33, pp. 1-5, 2017.

[37] R. P. Joshi, G. Negi, A. Kumar et al., "SNEDDS curcumin formulation leads to enhanced protection from pain and functional deficits associated with diabetic neuropathy: an insight into its mechanism for neuroprotection," Nanomedicine: Nanotechnology, Biology and Medicine, vol. 9, no. 6, pp. 776-785, 2013.

[38] S. Liu, Q. Li, and M. T. Zhang, "Curcumin ameliorates neuropathic pain by down-regulating spinal IL-1beta via suppressing astroglial NALP1 inflammasome and JAK2STAT3 signalling," Science Reports, vol. 6, p. 28956, 2016.

[39] L. Daulhac, C. Mallet, C. Courteix et al., "Diabetes-induced mechanical hyperalgesia involves spinal mitogen-activated protein kinase activation in neurons and microglia via N-methyl-D-aspartate-dependent mechanisms," Molecular Pharmacology, vol. 70, no. 4, pp. 1246-1254, 2006.

[40] Y.-J. Gao, Z.-Z. Xu, Y.-C. Liu, Y.-R. Wen, I. Decosterd, and R.-R. Ji, "The c-Jun N-terminal kinase 1 (JNK1) in spinal astrocytes is required for the maintenance of bilateral mechanical allodynia under a persistent inflammatory pain condition," Pain, vol. 148, no. 2, pp. 309-319, 2010.

[41] X. W. Wang, Q.-L. Hu, S. Fau - Mao-Ying, and Q. Mao-Ying Ql Fau - Li, "Activation of c-jun N-terminal kinase in spinal cord contributes to breast cancer induced bone pain in rats," Molecular Brain, vol. 5, no. 21, 2012.

[42] Y.-J. Gao, J.-K. Cheng, Q. Zeng et al., "Selective inhibition of JNK with a peptide inhibitor attenuates pain hypersensitivity and tumor growth in a mouse skin cancer pain model," Experimental Neurology, vol. 219, no. 1, pp. 146-155, 2009.

[43] R. Stavniichuk, H. Shevalye, H. Hirooka, J. L. Nadler, and I. G. Obrosova, "Interplay of sorbitol pathway of glucose metabolism, 12/15-lipoxygenase, and mitogen-activated protein kinases in the pathogenesis of diabetic peripheral neuropathy," Biochemical Pharmacology, vol. 83, no. 7, pp. 932-940, 2012.

[44] C.-P. Kuai, L.-J. Ju, P.-P. Hu, and F. Huang, "Corydalis saxicola alkaloids attenuate cisplatin-induced neuropathic pain by reducing loss of IENF and blocking TRPV1 activation," The American Journal of Chinese Medicine, vol. 48, no. 02, pp. 407-428, 2020. 